\title{
The Semianalytical Solutions for Stiff Systems of Ordinary Differential Equations by Using Variational Iteration Method and Modified Variational Iteration Method with Comparison to Exact Solutions
}

\author{
Mehmet Tarik Atay and Okan Kilic \\ Mathematics Department, Arts and Sciences Faculty, Nigde University, 51100 Nigde, Turkey \\ Correspondence should be addressed to Mehmet Tarik Atay; ataymt@nigde.edu.tr
}

Received 20 February 2013; Accepted 30 March 2013

Academic Editor: Safa Bozkurt Coskun

Copyright (C) 2013 M. T. Atay and O. Kilic. This is an open access article distributed under the Creative Commons Attribution License, which permits unrestricted use, distribution, and reproduction in any medium, provided the original work is properly cited.

\begin{abstract}
The Variational Iteration Method (VIM) and Modified Variational Iteration Method (MVIM) are used to find solutions of systems of stiff ordinary differential equations for both linear and nonlinear problems. Some examples are given to illustrate the accuracy and effectiveness of these methods. We compare our results with exact results. In some studies related to stiff ordinary differential equations, problems were solved by Adomian Decomposition Method and VIM and Homotopy Perturbation Method. Comparisons with exact solutions reveal that the Variational Iteration Method (VIM) and the Modified Variational Iteration Method (MVIM) are easier to implement. In fact, these methods are promising methods for various systems of linear and nonlinear stiff ordinary differential equations. Furthermore, VIM, or in some cases MVIM, is giving exact solutions in linear cases and very satisfactory solutions when compared to exact solutions for nonlinear cases depending on the stiffness ratio of the stiff system to be solved.
\end{abstract}

\section{Introduction}

Stiff system of ordinary differential equations (ODEs) can be seen in modeling various real-world problems [1]. Solving such a system of ODEs is important, and many numerical and analytical methods have been developed [1-17]. Classical numerical methods, such as finite difference and finite element methods are computationally expensive and are generally affected by round-off errors, which may give inaccurate solutions. For further details of stiff problems, the reader is referred to some previous studies in this field [117]. Examples are presented to illustrate the efficiency of the proposed approaches.

In this paper, Variation Iteration Method (VIM) and Modified Variation Iteration Method (MVIM) are proposed to obtain exact solutions of a stiff system of ODEs. This numerical scheme is based upon application of Variational Theory and is capable of finding the exact solutions of many linear differential equations.
The Variation Iteration Method was first proposed by He [18-23] and was successfully applied to autonomous ordinary differential equations by $\mathrm{He}$ [23], nonlinear polycrystalline solids [20], nonlinear partial differential equations, and other fields [19-25].

The purpose of this paper is to show the merits of VIM and MVIM in solving some stiff systems of ordinary differential equations. The Variation Iteration Method is useful for obtaining exact and approximate solutions of linear and nonlinear differential equations. There is no requirement for linearization or discretization and, hence, large computational work and round-off errors are avoided. The availability of computer symbolic packages gives a mathematical tool to perform some complicated manipulations and to carry out some modifications on a method for a specific problem easily. The results of the Variation Iteration Method (VIM) and Modified Variation Iteration Method (MVIM) are compared with the exact solutions of the problems to show the efficiency of these methods. 


\section{Variation Iteration Method}

In 1978, Inokuti et al. [26] introduced a general Lagrange multiplier method to solve nonlinear problems. In this method the solution of a mathematical problem with a linearization assumption is used as an initial approximation or trial function. To illustrate this method, consider the following form of a differential equation:

$$
L u(t)+N u(t)=g(t),
$$

where $L$ is a linear operator, $N$ is a nonlinear operator, and $g(t)$ is an inhomogeneous term.

He has modified the above mentioned method into an iteration procedure. According to the Variation Iteration Method, or more precisely, He's Variation Iteration Method [18-23], a correctional functional can be written in the following way:

$$
u_{n+1}(t)=u_{n+1}(t)+\int_{0}^{t} \lambda(\tau)\left(L u_{n}(\tau)+N \widetilde{u_{n}}(\tau)-g(\tau)\right) d \tau,
$$

where $\lambda$ is a general Lagrangian multiplier [19-23], which can be identified optimally via the Variational Theory, the subscript $n$ denotes the $n$th order approximation, and $\widetilde{\delta u_{n}}$ is considered as a restricted variation [19-23], that is, $\widetilde{\delta u_{n}}=0$. In this case, (2) is called a correction functional.

Also as it was indicated by Ramos [27], Inokuti et al.'s Variational Iteration and also He's Variation Iteration Method can be derived by means of Adjoint Operators, Green's Function, Integration by Parts, and the method of Weighted Residuals.

Based on this basic VIM and MVIM formulation knowledge, there are three different VIM algorithms given in [28]:

Algorithm 1.

$$
u_{n+1}(t)=u_{n}(t)+\int_{t_{0}}^{t} \lambda\left[L u_{n}(\tau)+N u_{n}(\tau)\right] d \tau,
$$

Algorithm 2.

$$
u_{n+1}(t)=u_{0}(t)+\int_{t_{0}}^{t} \lambda\left[N u_{n}(\tau)\right] d \tau,
$$

Algorithm 3.

$$
u_{n+2}(t)=u_{n+1}(t)+\int_{t_{0}}^{t} \lambda\left[N u_{n+1}(\tau)-N u_{n}(\tau)\right] d \tau
$$

Further details of these iteration procedures with examples can be found in [27].

\section{Stiffness of a System of Ordinary Differential Equations}

For a given system of ordinary differential equations, stiffness means a big difference in the time scales of the components in the vector solution. Some of well-known numerical methods and their procedures which are quite satisfactory in general can work unsatisfactorily on stiff problems. For a general formulation for stiff problems,

$$
\mathbf{y}^{\prime}=\mathbf{f}(x, \mathbf{y}), \quad \mathbf{y}\left(x_{0}\right)=\mathbf{y}_{0}, \quad a \leq x \leq b,
$$

where $\mathbf{f}(x, \mathbf{y})$ is defined and continuous in a region $D \subset$ $[a, b]$, that is either singular or stiff or both. The definition of stiffness is given in [15] and in [16] as follows.

The Initial Value Problem of (6) is said to be stiff over the finite interval for every $x \in[a, b]$, the eigenvalues $\left\{\lambda_{s}(x), s=\right.$ $1,2,3, \ldots, m\}$ of the Jacobian matrix satisfy the following conditions:

(a) $\operatorname{Re}\left(\lambda_{s}(x)\right)<0, \quad s=1,2,3, \ldots, m$,

(b) stiffness ratio $R=\frac{\max \left\|\operatorname{Re}\left(\lambda_{s}(x)\right)\right\|}{\min \left\|\operatorname{Re}\left(\lambda_{s}(x)\right)\right\|} \gg 1$,

$$
s=1,2,3, \ldots, m,
$$

where $\lambda_{s}$ are the eigenvalues of the Jacobian of the system.

\section{Applications}

In this section, Variation Iteration Method (VIM) and Modified Variational Method (MVIM) are applied to various stiff systems of ordinary differential equations.

4.1. Problem 1. The stiff differential equation of second order can be considered as a first example of this study elaborated in [29]. The problem is as follows:

$$
u^{\prime \prime}(t)=-20 u^{\prime}(t)-19 u(t)
$$

with initial conditions

$$
u(0)=2, \quad u^{\prime}(0)=-20
$$

Its exact analytical solution is $u(t)=e^{-19 t}+e^{-t}$.

Below, this second order ordinary differential equation can be written as a system of first order ordinary differential equations and the stiffness ratio for this system can be calculated as $R=19$ by using (7) and one can obtain the following system.

$$
\begin{gathered}
u^{\prime}=v, \\
v^{\prime}=-20 v-19 u .
\end{gathered}
$$

4.1.1. VIM Iteration Formulation. VIM iteration formulation for this system can be written as follows:

$$
\begin{gathered}
u_{n+1}(t)=u_{n}(t)+\int_{0}^{t} \lambda_{1}(s)\left[u_{n}^{\prime}(s)-v_{n}(s)\right] d s \\
v_{n+1}(t)=v_{n}(t)+\int_{0}^{t} \lambda_{2}(s)\left[v_{n}^{\prime}(s)+19 u_{n}(s)+20 v_{n}(s)\right] d s .
\end{gathered}
$$


Their Lagrange multipliers can be calculated as $\lambda_{1}(s)=-1$ and $\lambda_{2}(s)=-e^{20(s-t)}$. As an exemplary purpose, first and second iterations are given as follows for VIM formulation.

For $n=0$,

$$
u_{1}(t)=u_{0}(t)-\int_{0}^{t}\left[u_{0}^{\prime}(s)-v_{0}(s)\right] d s
$$

$$
v_{1}(t)=v_{0}(t)-\int_{0}^{t} e^{20(s-t)}\left[v_{0}^{\prime}(s)+19 u_{0}(s)+20 v_{0}(s)\right] d s
$$

For $n=1$,

$$
\begin{gathered}
u_{2}(t)=u_{1}(t)-\int_{0}^{t}\left[u_{1}^{\prime}(s)-v_{1}(s)\right] d s \\
v_{2}(t)=v_{1}(t)-\int_{0}^{t} e^{20(s-t)}\left[v_{1}^{\prime}(s)+19 u_{1}(s)+20 v_{1}(s)\right] d s
\end{gathered}
$$

By using the above written formulations, we can get the following approximations as

$$
\begin{gathered}
u_{1}(t)=2-20 t, \\
v_{1}(t)=-\frac{19}{10}-\frac{181 e^{-20 t}}{10}, \\
u_{2}(t)=\frac{219}{200}+\frac{181 e^{-20 t}}{200}-\frac{19 t}{10}, \\
v_{2}(t)=-\frac{57}{20}-\frac{343 e^{-20 t}}{20}+19 t .
\end{gathered}
$$

4.1.2. MVIM Iteration Formulation. MVIM iteration formulation for this system can be written as follows:

$$
\begin{array}{r}
m u_{n+1}(t)=m u_{n}(t)+\int_{0}^{t} \lambda_{1}(s)\left[m u_{n}^{\prime}(s)-m v_{n}(s)\right] d s \\
m v_{n+1}(t)=m v_{n}(t)+\int_{0}^{t} \lambda_{2}(s)\left[m v_{n}^{\prime}(s)+19 m u_{n+1}(s)\right. \\
\left.+20 m v_{n}(s)\right] d s
\end{array}
$$

For $n=0$,

$$
\begin{array}{r}
m u_{1}(t)=m u_{0}(t)-\int_{0}^{t}\left[m u_{0}^{\prime}(s)-m v_{0}(s)\right] d s \\
m v_{1}(t)=m v_{0}(t)-\int_{0}^{t} e^{20(s-t)}\left[m v_{0}^{\prime}(s)+19 m u_{1}(s)\right. \\
\left.+20 m v_{0}(s)\right] d s .
\end{array}
$$

For $n=1$,

$$
\begin{gathered}
m u_{2}(t)=m u_{1}(t)-\int_{0}^{t}\left[m u_{1}^{\prime}(s)-m v_{1}(s)\right] d s, \\
m v_{2}(t)=m v_{1}(t)-\int_{0}^{t} e^{20(s-t)}\left[m v_{1}^{\prime}(s)+19 m u_{2}(s)\right. \\
\left.+20 m v_{1}(s)\right] d s,
\end{gathered}
$$

or as computed iterations as follows:

$$
\begin{gathered}
m u_{1}(t)=2-20 t \\
m v_{1}(t)=-\frac{57}{20}-\frac{343 e^{-20 t}}{20}+19 t \\
m u_{2}(t)=\frac{457}{400}+\frac{343 e^{-20 t}}{400}-\frac{57 t}{20}+\frac{19 t^{2}}{2}, \\
m v_{2}(t)=-\frac{10127}{8000}-\frac{14987 e^{-20 t}}{8000}+\frac{361 t}{100} \\
-\frac{6517 t}{400} e^{-20 t}-\frac{361 t^{2}}{40} .
\end{gathered}
$$

In the interval of $0 \leq t \leq 1$ VIM and MVIM results are obtained for the 15th iteration. These results are compared with the exact result of the stiff ordinary differential equation for error analysis purpose in Tables 1 and 2.

4.2. Problem 2. This stiff ordinary differential equations system example is presented in [9] and has the following form:

$$
\left(\begin{array}{l}
u^{\prime}(t) \\
v^{\prime}(t)
\end{array}\right)=\left(\begin{array}{cc}
-10 & 1 \\
1 & -2
\end{array}\right)\left(\begin{array}{l}
u(t) \\
v(t)
\end{array}\right)
$$

with initial conditions

$$
\left(\begin{array}{l}
u(0) \\
v(0)
\end{array}\right)=\left(\begin{array}{l}
2 \\
8
\end{array}\right)
$$

The stiffness ratio $R$ of the system is $R=5.393540395$ and can be easily found by using (7). The exact solutions of this stiff ordinary differential equations system can be obtained by Laplace transform method as follows:

$$
\begin{gathered}
u_{E}(t)=e^{(-6-\sqrt{17}) t}+e^{(-6+\sqrt{17}) t} \\
v_{E}(t)=\frac{(-17+4 \sqrt{17}) e^{(-6-\sqrt{17}) t}+(17+4 \sqrt{17}) e^{(-6+\sqrt{17}) t}}{\sqrt{17}} .
\end{gathered}
$$

4.2.1. VIM Iteration Formulation. We can construct the following correction functionals for vector $\left(\begin{array}{c}u(t) \\ v(t)\end{array}\right)$ as follows:

$$
\begin{gathered}
u_{n+1}(t)=u_{n}(t)+\int_{0}^{t} \lambda_{1}(s)\left[u_{n}^{\prime}(s)+10 u_{n}(s)-\widetilde{v}_{n}(s)\right] d s, \\
v_{n+1}(t)=v_{n}(t)+\int_{0}^{t} \lambda_{2}(s)\left[v_{n}^{\prime}(s)-\widetilde{u}_{n}(s)+2 v_{n}(s)\right] d s
\end{gathered}
$$


TABLE 1: Values for $u(t)$ for the fifteenth iteration by Variational Iteration Method (VIM) and Modified Variational Iteration Method (MVIM) compared with exact values of $u(t)$.

\begin{tabular}{lccc}
\hline$t$ & $\operatorname{VIM} u_{15}$ & MVIM $u_{15}$ & Exact $u$ \\
\hline 0 & 2 & 2 & 2 \\
0.1 & 1.0544060372585944 & 1.0544060372585946 & 1.0544060372585946 \\
0.2 & 0.8411015249341647 & 0.8411015249341475 & 0.8411015249341474 \\
0.3 & 0.744164186144312 & 0.744164186139189 & 0.7441641861391891 \\
0.4 & 0.6708204977097115 & 0.6708204974690799 & 0.6708204974690799 \\
0.5 & 0.6066055157577269 & 0.6066055115425211 & 0.6066055115425211 \\
0.6 & 0.5488228716973902 & 0.5488228315788688 & 0.548822831578869 \\
0.7 & 0.49658723146673706 & 0.49658697828461873 & 0.4965869782846189 \\
0.8 & 0.44933040627822074 & 0.4493292145688589 & 0.44932921456885877 \\
0.9 & 0.40657420615772905 & 0.40656969720030467 & 0.40656969720030467 \\
1 & 0.36789386847489264 & 0.36787944677423856 & 0.3678794467742388 \\
\hline
\end{tabular}

where $\lambda_{1}$ and $\lambda_{2}$ are general Lagrange multipliers and $\tilde{u}_{n}$ and $\widetilde{v}_{n}$ denote restricted variations. In this respect, we have $\delta \widetilde{u}_{n}=0$ and $\widetilde{\delta v_{n}}=0$. In order to construct correction functional, (24) should be elaborated as follows:

$$
\begin{aligned}
\delta u_{n+1}(t)= & \delta u_{n}(t)+\delta \int_{0}^{t} \lambda_{1}(s)\left[u_{n}^{\prime}(s)+10 u_{n}(s)-\widetilde{v}_{n}(s)\right] d s \\
= & \delta u_{n}(t)+\delta \int_{0}^{t} \lambda_{1}(s)\left[u_{n}^{\prime}(s)+10 u_{n}(s)\right] d s=0 \\
= & \delta u_{n}(s)+\lambda_{1} \cdot \delta u_{n}(s) \|_{0}^{t} \\
& +\int_{0}^{t}\left(10 \lambda_{1}(s)-\lambda_{1}^{\prime}(s)\right) \delta u_{n}(s) d s=0 .
\end{aligned}
$$

From here, by using stationary conditions obtained from (25), following iteration formulas,

$$
\begin{aligned}
& u_{n+1}(t)=u_{n}(t)+\int_{0}^{t} \lambda_{1}(s)\left[u_{n}^{\prime}(s)+10 u_{n}(s)-v_{n}(s)\right] d s, \\
& v_{n+1}(t)=v_{n}(t)+\int_{0}^{t} \lambda_{2}(s)\left[v_{n}^{\prime}(s)-u_{n}(s)+2 v_{n}(s)\right] d s,
\end{aligned}
$$

and general Lagrange multipliers can be obtained as follows:

$$
\lambda_{1}(s)=-e^{10(s-t)}, \quad \lambda_{2}(s)=-e^{2(s-t)}
$$

for this problem. As an example, first and second VIM iterations of the problem given in (21)-(22) can easily be found by using (26)-(27) together.

For $n=0$,

$$
\begin{gathered}
u_{1}(t)=u_{0}(t)-\int_{0}^{t} e^{10(s-t)}\left[u_{0}^{\prime}(s)+10 u_{0}(s)-v_{0}(s)\right] d s \\
v_{1}(t)=v_{0}(t)-\int_{0}^{t} e^{2(s-t)}\left[v_{0}^{\prime}(s)-u_{0}(s)+2 v_{0}(s)\right] d s
\end{gathered}
$$

TABLE 2: Absolute error values for $u(t)$ for the fifteenth iteration by Variational Iteration Method (VIM) and Modified Variational Iteration Method (MVIM).

\begin{tabular}{lcc}
\hline$t$ & $\left|u(t)-u_{15}(t)\right|$ & $\left|u(t)-m u_{15}(t)\right|$ \\
\hline 0 & 0 & 0 \\
0.1 & $2.2887169047990097 * 10^{-16}$ & $1.7412077124868676 * 10^{-17}$ \\
0.2 & $1.7246525930471324 * 10^{-14}$ & $1.0492619227905604 * 10^{-16}$ \\
0.3 & $5.12282328102992 * 10^{-12}$ & $1.1491517324386781 * 10^{-17}$ \\
0.4 & $2.4063157065892793 * 10^{-10}$ & $7.613359770026226 * 10^{-17}$ \\
0.5 & $4.215205883992268 * 10^{-9}$ & $5.942063179931005 * 10^{-17}$ \\
0.6 & $4.0118521299307985 * 10^{-8}$ & $2.1285853261205842 * 10^{-16}$ \\
0.7 & $2.531821182757271 * 10^{-7}$ & $9.624496566471713 * 10^{-17}$ \\
0.8 & $1.1917093621013897 * 10^{-6}$ & $5.238729372178397 * 10^{-17}$ \\
0.9 & $4.508957424324139 * 10^{-6}$ & $1.0421893383016911 * 10^{-17}$ \\
1 & 0.000014421700653924341 & $2.388226335442445 * 10^{-16}$ \\
\hline
\end{tabular}

or, as a computed result for this first iteration,

$$
u_{1}(t)=\frac{4}{5}+\frac{6 e^{-10 t}}{5}, \quad v_{1}(t)=1+7 e^{-2 t}
$$

For $n=1$,

$$
\begin{gathered}
u_{2}(t)=u_{1}(t)-\int_{0}^{t} e^{10(s-t)}\left[u_{1}^{\prime}(s)+10 u_{1}(s)-v_{1}(s)\right] d s, \\
v_{2}(t)=v_{1}(t)-\int_{0}^{t} e^{2(s-t)}\left[v_{1}^{\prime}(s)-u_{1}(s)+2 v_{1}(s)\right] d s,
\end{gathered}
$$

or, as a computed result for this second iteration,

$$
\begin{aligned}
& u_{2}(t)=\frac{1}{10}+\frac{41 e^{-10 t}}{40}+\frac{7 e^{-2 t}}{8} \\
& v_{2}(t)=\frac{2}{5}-\frac{3 e^{-10 t}}{20}+\frac{31 e^{-2 t}}{4} .
\end{aligned}
$$

VIM iteration of this problem is conducted up to 15 iterations and results and related errors are given in Tables 3, 4, 5, and 6 for comparison purposes. 
TABLE 3: Values for $u(t)$ for the fifteenth iteration by Variational Iteration Method (VIM) and Modified Variational Iteration Method (MVIM) compared with exact values of $u(t)$.

\begin{tabular}{cccc}
\hline$t$ & $\operatorname{VIM} u_{15}$ & MVIM $u_{15}$ & Exact $u$ \\
\hline 0 & 2 & 2 & 2 \\
1 & 0.15310487138764364 & 0.15310487138763976 & 0.15310487138763973 \\
2 & 0.02342881322120863 & 0.02342881321791905 & 0.02342881321791905 \\
3 & 0.0035861247768383723 & 0.0035861247256523954 & 0.003586124725652396 \\
4 & 0.0005489094046572347 & 0.0005489092132974475 & 0.0005489092132974475 \\
5 & 0.00008401900780327056 & 0.00008401864059355628 & 0.00008401864059355636 \\
\hline
\end{tabular}

TABLE 4: Values for $v(t)$ for the fifteenth iteration by Variational Iteration Method (VIM) and Modified Variational Iteration Method (MVIM) compared with exact values of $v(t)$.

\begin{tabular}{lccc}
\hline$t$ & VIM $v_{15}$ & MVIM $v_{15}$ & Exact $v$ \\
\hline 0 & 8 & 7.9999999999999999 & 8.000000000000002 \\
1 & 1.2433560287025747 & 1.2433560287025707 & 1.243356028702570 \\
2 & 0.1903147111678612 & 0.19031471116472698 & 0.19031471116472704 \\
3 & 0.02913046998493388 & 0.029130469932580193 & 0.0291304699325802 \\
4 & 0.004458847727178969 & 0.004458847518489839 & 0.00445884751848984 \\
5 & 0.0006824927138448505 & 0.0006824922920622653 & 0.000682492292062266 \\
\hline
\end{tabular}

TABLE 5: Absolute Error Values for $u(t)$ for the fifteenth iteration by Variational Iteration Method (VIM) and Modified Variational Iteration Method (MVIM).

\begin{tabular}{ccc}
\hline$t$ & $\left|u(t)-u_{15}(t)\right|$ & $\left|u(t)-m u_{15}(t)\right|$ \\
\hline 0 & 0 & 0 \\
1 & $3.9361078957821094 * 10^{-15}$ & $4.8262061805908646 * 10^{-17}$ \\
2 & $3.289578231666611 * 10^{-12}$ & $7.555517345896108 * 10^{-19}$ \\
3 & $5.1185976684536014 * 10^{-11}$ & $3.215333759054874 * 10^{-19}$ \\
4 & $1.9135978719018548 * 10^{-10}$ & $1.2307620334176499 * 10^{-20}$ \\
5 & $3.672097141931768 * 10^{-10}$ & $7.506581340857471 * 10^{-20}$ \\
\hline
\end{tabular}

TABLE 6: Absolute Error Values for $v(t)$ for the fifteenth iteration by Variational Iteration Method (VIM) and Modified Variational Iteration Method (MVIM).

\begin{tabular}{ccc}
\hline$t$ & $\left|v(t)-v_{15}(t)\right|$ & $\left|v(t)-m v_{15}(t)\right|$ \\
\hline 0 & $1.7763568394002505 * 10^{-15}$ & $2.6229018956769323 * 10^{-15}$ \\
1 & $3.796171276632121 * 10^{-15}$ & $6.699623261226515 * 10^{-17}$ \\
2 & $3.1341844809566755 * 10^{-12}$ & $5.768933781415289 * 10^{-17}$ \\
3 & $5.235368044577589 * 10^{-11}$ & $7.028651272175357 * 10^{-18}$ \\
4 & $2.086891291014619 * 10^{-10}$ & $1.3107024221759996 * 10^{-18}$ \\
5 & $4.2178258450234333 * 10^{-10}$ & $6.661330967823222 * 10^{-19}$ \\
\hline
\end{tabular}

4.2.2. MVIM Iteration Formulation. One has

$$
\begin{aligned}
m u_{n+1}(t)= & m u_{n}(t) \\
& +\int_{0}^{t} \lambda_{1}(s)\left[m u_{n}^{\prime}(s)+10 m u_{n}(s)-m v_{n}(s)\right] d s, \\
m v_{n+1}(t)= & m v_{n}(t) \\
& +\int_{0}^{t} \lambda_{2}(s)\left[m v_{n}^{\prime}(s)-m u_{n+1}(s)+2 m v_{n}(s)\right] d s .
\end{aligned}
$$

and general Lagrange multipliers can be obtained as follows:

$$
\lambda_{1}(s)=-e^{10(s-t)}, \quad \lambda_{2}(s)=-e^{2(s-t)}
$$

for this problem. As an example, first and second MVIM iterations of the problem given in (21)-(22) can easily be found by using (32)-(33) together.

For $n=0$,

$$
\begin{aligned}
m u_{1}(t)= & m u_{0}(t) \\
& -\int_{0}^{t} e^{10(s-t)}\left[m u_{0}^{\prime}(s)+10 m u_{0}(s)-m v_{0}(s)\right] d s,
\end{aligned}
$$

$m v_{1}(t)=m v_{0}(t)$

$$
-\int_{0}^{t} e^{2(s-t)}\left[m v_{0}^{\prime}(s)-m u_{1}(s)+2 m v_{0}(s)\right] d s,
$$

or, as a computed result for this first iteration,

$$
\begin{gathered}
m u_{1}(t)=\frac{4}{5}+\frac{6 e^{-10 t}}{5} \\
m v_{1}(t)=\frac{2}{5}-\frac{3 e^{-10 t}}{20}+\frac{31 e^{-2 t}}{4}
\end{gathered}
$$

For $n=1$,

$$
\begin{aligned}
m u_{2}(t)= & m u_{1}(t) \\
& -\int_{0}^{t} e^{10(s-t)}\left[m u_{1}^{\prime}(s)+10 m u_{1}(s)-m v_{1}(s)\right] d s, \\
m v_{2}(t)= & m v_{1}(t) \\
& -\int_{0}^{t} e^{2(s-t)}\left[m v_{1}^{\prime}(s)-m u_{2}(s)+2 m v_{1}(s)\right] d s,
\end{aligned}
$$


or, as a computed result for this second iteration,

$$
\begin{gathered}
m u_{2}(t)=\frac{1}{25}+\frac{793 e^{-10 t}}{800}+\frac{31 e^{-2 t}}{32}-\frac{3}{20} e^{-10 t} t \\
m v_{2}(t)= \\
\frac{1}{50}-\frac{389 e^{-10 t}}{3200}+\frac{1037 e^{-2 t}}{128} \\
+\frac{3}{160} e^{-10 t} t+\frac{31}{32} e^{-2 t} t
\end{gathered}
$$

MVIM iteration of this problem is conducted up to 15 iterations and results and related errors are given in Tables 3-6 for comparison purposes.

4.3. Problem 3. Let us consider the following stiff ordinary differential equations system with stiffness ratio as $R=1000$ as an our next example which is given in [30]

$$
\left(\begin{array}{l}
u^{\prime}(t) \\
v^{\prime}(t)
\end{array}\right)=\left(\begin{array}{cc}
998 & 1998 \\
-999 & -1999
\end{array}\right)\left(\begin{array}{l}
u(t) \\
v(t)
\end{array}\right)
$$

with initial conditions as follows:

$$
\left(\begin{array}{l}
u(0) \\
v(0)
\end{array}\right)=\left(\begin{array}{l}
1 \\
1
\end{array}\right)
$$

with exact solutions:

$$
\begin{gathered}
u(t)=4 e^{-t}-3 e^{-1000 t}, \\
v(t)=-2 e^{-t}+3 e^{-1000 t}
\end{gathered}
$$

4.3.1. VIM Iteration Formulation. VIM iteration formulation for this system can be written as follows:

$$
\begin{aligned}
u_{n+1}(t)= & u_{n}(t) \\
& +\int_{0}^{t} \lambda_{1}(s)\left[u_{n}^{\prime}(s)-998 u_{n}(s)-1998 v_{n}(s)\right] d s, \\
v_{n+1}(t)= & v_{n}(t) \\
& +\int_{0}^{t} \lambda_{2}(s)\left[v_{n}^{\prime}(s)+999 u_{n}(s)+1999 v_{n}(s)\right] d s .
\end{aligned}
$$

Their Lagrange multipliers can be calculated as $\lambda_{1}(s)=$ $-e^{-998(s-t)}$ and $\lambda_{2}(s)=-e^{1999(s-t)}$. As an exemplary purpose, first and second iterations are given as follows for VIM formulation.

The stiffness ratio of the system $(R)$ is equal to 1000 .
For $n=0$,

$$
\begin{aligned}
u_{1}(t)= & u_{0}(t) \\
& -\int_{0}^{t} e^{-998(s-t)}\left[u_{0}^{\prime}(s)-998 u_{0}(s)-1998 v_{0}(s)\right] d s, \\
v_{1}(t)= & v_{0}(t) \\
& -\int_{0}^{t} e^{-1999(s-t)}\left[v_{0}^{\prime}(s)+999 u_{0}(s)+1999 v_{0}(s)\right] d s .
\end{aligned}
$$

For $n=1$,

$$
\begin{aligned}
u_{2}(t)= & u_{1}(t) \\
& -\int_{0}^{t} e^{-998(s-t)}\left[u_{1}^{\prime}(s)-998 u_{1}(s)-1998 v_{1}(s)\right] d s, \\
v_{2}(t)= & v_{1}(t) \\
& -\int_{0}^{t} e^{-1999(s-t)}\left[v_{1}^{\prime}(s)+999 u_{1}(s)+1999 v_{1}(s)\right] d s .
\end{aligned}
$$

By using the above written formulations, we can get the following approximations as

$$
\begin{gathered}
u_{1}(t)=-\frac{999}{499}+\frac{1498 e^{998 t}}{499}, \\
v_{1}(t)=-\frac{999}{1999}+\frac{2998 e^{-1999 t}}{1999}, \\
u_{2}(t)=\frac{998001}{997501}-\frac{5996 e^{-1999 t}}{5997}+\frac{1496 e^{998 t}}{1497}, \\
v_{2}(t)=\frac{998001}{997501}+\frac{5998 e^{-1999 t}}{5997}-\frac{1498 e^{998 t}}{1497} .
\end{gathered}
$$

4.3.2. MVIM Iteration Formulation. MVIM iteration formulation for this system can be written as follows:

$$
\begin{aligned}
m u_{n+1}(t)= & m u_{n}(t) \\
& +\int_{0}^{t} \lambda_{1}(s)\left[m u_{n}^{\prime}(s)-998 m u_{n}(s)\right. \\
m v_{n+1}(t)= & \left.-1998 m v_{n}(s)\right] d s, \\
& +\int_{0}^{t} \lambda_{2}(s)\left[m v_{n}^{\prime}(s)+999 m u_{n+1}(s)\right. \\
& \left.+1999 m v_{n}(s)\right] d s .
\end{aligned}
$$


For $n=0$,

$$
\begin{array}{r}
m u_{1}(t)=m u_{0}(t)-\int_{0}^{t} e^{-998(s-t)}\left[m u_{0}^{\prime}(s)-998 m u_{0}(s)\right. \\
\left.-1998 m v_{0}(s)\right] d s
\end{array}
$$

$m v_{1}(t)=m v_{0}(t)$

$$
\begin{gathered}
-\int_{0}^{t} e^{-1999(s-t)}\left[m v_{0}^{\prime}(s)+999 m u_{1}(s)\right. \\
\left.+1999 m v_{0}(s)\right] d s .
\end{gathered}
$$

For $n=1$,

$$
\begin{aligned}
m u_{2}(t)= & m u_{1}(t) \\
& -\int_{0}^{t} e^{-998(s-t)}\left[m u_{1}^{\prime}(s)-998 m u_{1}(s)\right. \\
& \left.-1998 m v_{1}(s)\right] d s \\
m v_{2}(t)= & m v_{1}(t) \\
& -\int_{0}^{t} e^{-1999(s-t)}\left[m v_{1}^{\prime}(s)+999 m u_{2}(s)\right. \\
& \left.+1999 m v_{1}(s)\right] d s
\end{aligned}
$$

or as computed iterations as follows:

$$
\begin{aligned}
m u_{1}(t)=-\frac{999}{499}+\frac{1498 e^{998 t}}{499} & \\
m v_{1}(t)= & \frac{998001}{997501}+\frac{5998 e^{-1999 t}}{5997}-\frac{1498 e^{998 t}}{1497}, \\
m u_{2}(t)= & -\frac{997002999}{497752999}-\frac{11996 e^{-1999 t}}{17991} \\
& +\frac{8224022 e^{998 t}}{2241009}-\frac{997668 t}{499} e^{998 t}, \\
m v_{2}(t)= & \frac{996005996001}{995008245001}+\frac{155864026 e^{-1999 t}}{107892027} \\
& -\frac{9719026 e^{998 t}}{6723027}+\frac{1331556 t}{1999} e^{-1999 t} \\
& +\frac{332556 t}{499} e^{998 t} \cdot
\end{aligned}
$$

The results and related error analysis in the interval of $0 \leq t \leq$ 0.001 are obtained by using VIM and MVIM. The results are given in Tables 7, 8, 9, and 10.

4.4. Problem 4. Current stiff ordinary differential equations system example is elaborated in [8] and has the following form:

$$
\begin{gathered}
u^{\prime}(t)=0.01-(0.01+u(t)+v(t)) \\
\times\left(u^{2}(t)+1001 u(t)+1001\right), \\
v^{\prime}(t)=0.01-(0.01+u(t)+v(t))\left(1+v^{2}\right)
\end{gathered}
$$

with initial conditions

$$
\begin{aligned}
& u(0)=0 \\
& v(0)=0 .
\end{aligned}
$$

4.4.1. VIM Iteration Formulation. By using VIM iteration formula for this problem,

$$
\begin{aligned}
& u_{n+1}(t)=u_{n}(t)+\int_{0}^{t} \lambda_{1}(s) \\
& \times\left[u_{n}^{\prime}(s)-0.01\right. \\
& +\left(0.01+u_{n}(s)+v_{n}(s)\right) \\
& \left.\times\left(u_{n}^{2}(t)+1001 u_{n}(t)+1001\right)\right] d s, \\
& v_{n+1}(t)=v_{n}(t)+\int_{0}^{t} \lambda_{2}(s) \\
& \times\left[v_{n}^{\prime}(s)-0.01+\left(0.01+u_{n}(s)+v_{n}(s)\right)\right. \\
& \left.\times\left(1+v_{n}^{2}(s)\right)\right] d s
\end{aligned}
$$

are obtained with general Lagrange multipliers of this problem

$$
\lambda_{1}(s)=-e^{-1011.01(s-t)}, \quad \lambda_{2}(s)=-e^{s-t} .
$$

Furthermore, by using (7), it can be calculated that the Stiffness Ratio $R$ of this current nonlinear ordinary differential equations system is 102312 , so $R=102312$ for this system. It should be noted that this current $R$ value is much higher than the previous example's $R$ value. 
TABLE 7: Values for $u(t)$ for the fifteenth iteration by Variational Iteration Method (VIM) and Modified Variational Iteration Method (MVIM) compared with exact values of $u(t)$.

\begin{tabular}{lccc}
\hline$t$ & $\operatorname{VIM} u_{15}$ & MVIM $u_{15}$ & Exact $u$ \\
\hline 0 & 1.0000000000000009 & 0.9999999999999991 & 1 \\
0.0001 & 1.2850877658914557 & 1.2850877658914535 & 1.2850877658914546 \\
0.0002 & 1.5430078207607218 & 1.54300782076072 & 1.5430078207607214 \\
0.0003 & 1.7763455179368497 & 1.7763455179368466 & 1.7763455179368477 \\
0.0004 & 1.9874401818504213 & 1.9874401818504188 & 1.98744018185042 \\
0.0005 & 2.178408520778776 & 2.178408520778775 & 2.1784085207787767 \\
0.0006 & 2.3511658115739342 & 2.351165811573942 & 2.3511658115739427 \\
0.0007 & 2.5074450683970526 & 2.5074450683971445 & 2.5074450683971445 \\
0.0008 & 2.648814387306315 & 2.648814387307067 & 2.64881438730707 \\
0.0009 & 2.776692640287484 & 2.7766926402923118 & 2.776692640292312 \\
0.001 & 2.8923636757938955 & 2.8923636758191704 & 2.892363675819173 \\
\hline
\end{tabular}

TABLE 8: Values for $v(t)$ for the fifteenth iteration by Variational Iteration Method (VIM) and Modified Variational Iteration Method (MVIM) compared with exact values of $v(t)$.

\begin{tabular}{lccc}
\hline$t$ & VIM $v_{15}$ & MVIM $v_{15}$ & Exact $v$ \\
\hline 0 & 0.9999999999999998 & 1.0000000000000004 & 1 \\
0.0001 & 0.7147122441082119 & 0.7147122441082117 & 0.7147122441082121 \\
0.0002 & 0.4565922192366122 & 0.4565922192366125 & 0.4565922192366121 \\
0.0003 & 0.2230545720541527 & 0.22305457205415302 & 0.22305457205415302 \\
0.0004 & 0.011759978128248435 & 0.011759978128248725 & 0.011759978128248916 \\
0.0005 & -0.1794082708204386 & -0.17940827082043712 & -0.1794082708204383 \\
0.0006 & -0.35236545164593536 & -0.35236545164593086 & -0.3523654516459318 \\
0.0007 & -0.5088445785114996 & -0.508844578511458 & -0.5088445785114579 \\
0.0008 & -0.6504137474780325 & -0.6504137474777034 & -0.6504137474777028 \\
0.0009 & -0.7784918305373185 & -0.7784918305352573 & -0.7784918305352575 \\
0.001 & -0.894362676163021 & -0.8943626761524226 & -0.894362676152423 \\
\hline
\end{tabular}

TABLe 9: Absolute Error Values for $u(t)$ for the fifteenth iteration by Variational Iteration Method (VIM) and Modified Variational Iteration Method (MVIM).

\begin{tabular}{lcc}
\hline$t$ & $\left|u(t)-u_{15}(t)\right|$ & $\left|u(t)-m u_{15}(t)\right|$ \\
\hline 0 & $8.881784197001252 * 10^{-16}$ & $8.881784197001252 * 10^{-16}$ \\
0.0001 & $1.4107638668381384 * 10^{-15}$ & $1.63927337215093 * 10^{-15}$ \\
0.0002 & $4.874572967494828 * 10^{-16}$ & $3.636731723871602 * 10^{-16}$ \\
0.0003 & $1.762479051592436 * 10^{-15}$ & $1.6310915359790852 * 10^{-15}$ \\
0.0004 & $1.7208456881689926 * 10^{-15}$ & $2.045543910037667 * 10^{-15}$ \\
0.0005 & $5.412337245047638 * 10^{-16}$ & $2.501877828173238 * 10^{-15}$ \\
0.0006 & $8.18789480661053 * 10^{-15}$ & $2.9766770645589524 * 10^{-16}$ \\
0.0007 & $9.009459844833145 * 10^{-14}$ & $1.3595895242968226 * 10^{-16}$ \\
0.0008 & $7.55506768257419 * 10^{-13}$ & $2.9303816317938214 * 10^{-15}$ \\
0.0009 & $4.828359934094806 * 10^{-12}$ & $1.551710149261254 * 10^{-15}$ \\
0.001 & $2.5278001913875414 * 10^{-11}$ & $2.39044894989604 * 10^{-15}$ \\
\hline
\end{tabular}

TABLE 10: Absolute Error Values for $v(t)$ for the fifteenth iteration by Variational Iteration Method (VIM) and Modified Variational Iteration Method (MVIM).

\begin{tabular}{lcc}
\hline$t$ & $\left|v(t)-v_{15}(t)\right|$ & $\left|v(t)-m v_{15}(t)\right|$ \\
\hline 0 & $2.220446049250313 * 10^{-16}$ & $4.440892098500626 * 10^{-16}$ \\
0.0001 & $4.437097390896927 * 10^{-16}$ & $2.5681613855185254 * 10^{-16}$ \\
0.0002 & $8.370040771588094 * 10^{-17}$ & $4.270630030588457610^{-16}$ \\
0.0003 & $1.7607443281164592 * 10^{-16}$ & $2.1077031846439075 * 10^{-16}$ \\
0.0004 & $4.891920202254596 * 10^{-16}$ & $1.8985618825906542 * 10^{-16}$ \\
0.0005 & $5.412337245047638 * 10^{-16}$ & $9.454598697911445 * 10^{-16}$ \\
0.0006 & $3.802513859341161 * 10^{-15}$ & $7.902156712183764 * 10^{-16}$ \\
0.0007 & $4.1175396425785493 * 10^{-14}$ & $1.22514845490862 * 10^{-17}$ \\
0.0008 & $3.295558270721699 * 10^{-13}$ & $1.3088353100973449 * 10^{-15}$ \\
0.0009 & $2.0607682227336 * 10^{-12}$ & $4.040821496853475 * 10^{-16}$ \\
0.001 & $1.0597828170588741 * 10^{-11}$ & $1.2790333028811496 * 10^{-15}$ \\
\hline
\end{tabular}

For $n=0$, the first iteration will be as follows:

$$
\begin{aligned}
u_{1}(t)= & u_{0}(t) \\
& -\int_{0}^{t} e^{-1011.01(s-t)}
\end{aligned}
$$

$$
\begin{aligned}
& \times\left[u_{0}^{\prime}(s)-0.01\right. \\
& \quad+\left(0.01+u_{0}(s)+v_{0}(s)\right) \\
& \left.\quad \times\left(u_{0}^{2}(t)+1001 u_{0}(t)+1001\right)\right] d s,
\end{aligned}
$$




$$
\begin{aligned}
& v_{1}(t)=v_{0}(t) \\
& \qquad \begin{aligned}
-\int_{0}^{t} e^{s-t}[ & v_{0}^{\prime}(s)-0.01 \\
& +\left(0.01+u_{0}(s)+v_{0}(s)\right) \\
& \left.\times\left(1+v_{0}^{2}(s)\right)\right] d s,
\end{aligned}
\end{aligned}
$$

or, as a computed result for this first iteration,

$$
\begin{gathered}
u_{1}(t)=-0.00989109900000989 \\
+0.00989109900000989 e^{-1011.01 t}, \\
v_{1}(t)=0 .
\end{gathered}
$$

For $n=1$, the second iteration will be as follows:

$$
\begin{aligned}
& u_{2}(t)=u_{1}(t) \\
& -\int_{0}^{t} e^{-1011.01(s-t)} \\
& \times\left[u_{1}^{\prime}(s)-0.01\right. \\
& \quad+\left(0.01+u_{1}(s)+v_{1}(s)\right) \\
& \left.\quad \times\left(u_{1}^{2}(t)+1001 u_{1}(t)+1001\right)\right] d s,
\end{aligned}
$$

$v_{2}(t)=v_{1}(t)$

$$
\begin{aligned}
-\int_{0}^{t} e^{s-t}\left[v_{1}^{\prime}(s)-0.01\right. \\
+ \\
+\left(0.01+u_{1}(s)+v_{1}(s)\right) \\
\left.\quad \times\left(1+v_{1}^{2}(s)\right)\right] d s,
\end{aligned}
$$

or, as a computed result for this second iteration,

$$
\begin{aligned}
u_{2}(t)= & -0.009987964198100541 \\
& +4.785730068611516 \\
& \times 10^{-10} e^{-3033.0299999999993 t}+\cdots, \\
v_{2}(t)= & 0.009891099000000989 \\
& +0.000009793070365649738 e^{-1011.01 t} \\
& -0.00990089207037554 e^{-t} .
\end{aligned}
$$

4.4.2. MVIM Iteration Formulation. From Modified Variation Iteration Method (MVIM), the following iteration formulation can be obtained for (51)-(52):

$$
\begin{aligned}
m u_{n+1}(t)= & m u_{n}(t) \\
+\int_{0}^{t} \lambda_{1}(s)[ & m u_{n}^{\prime}(s)-0.01 \\
& +\left(0.01+m u_{n}(s)+m v_{n}(s)\right) \\
& \times\left(m u_{n}^{2}(t)+1001 m u_{n}(t)\right. \\
& +1001)] d s, \\
m v_{n+1}(t)=m v_{n}(t) \quad & \\
+\int_{0}^{t} \lambda_{2}(s)[ & m v_{n}^{\prime}(s)-0.01 \\
& +\left(0.01+m u_{n+1}(s)+m v_{n}(s)\right) \\
& \left.\times\left(1+m v_{n}^{2}(s)\right)\right] d s .
\end{aligned}
$$

Here again the same general Lagrange multipliers are used for calculations, for example,

$$
\begin{aligned}
& \lambda_{1}(s)=-e^{-1011.01(s-t)}, \quad \lambda_{2}(s)=-e^{s-t} . \\
& \text { For } n=0 \text {, } \\
& m u_{1}(t)=m u_{0}(t) \\
& -\int_{0}^{t} e^{-1011.01(s-t)}\left[m u_{0}^{\prime}(s)-0.01\right. \\
& +\left(0.01+m u_{0}(s)+m v_{0}(s)\right) \\
& \times\left(m u_{0}^{2}(t)+1001 m u_{0}(t)\right. \\
& +1001)] d s, \\
& m v_{1}(t)=m v_{0}(t) \\
& -\int_{0}^{t} e^{s-t}\left[m v_{0}^{\prime}(s)-0.01\right. \\
& +\left(0.01+m u_{1}(s)+m v_{0}(s)\right) \\
& \left.\times\left(1+m v_{0}^{2}(s)\right)\right] d s,
\end{aligned}
$$

or, as a computed result for this first iteration,

$$
\begin{aligned}
m u_{1}(t)= & -0.00989109900000989 \\
& +0.00989109900000989 e^{-1011.01 t} \\
m v_{1}(t)= & 0.00989109900000989 \\
& +0.000009793070365649738 e^{-1011.01 t} \\
& -0.00989109900000989 e^{-t}-\cdots
\end{aligned}
$$


TABLE 11: Values for $u(t)$ for the second iteration by Variational Iteration Method (VIM) and Modified Variational Iteration Method (MVIM) compared with exact values of $u(t)$ in $0.0001 \leq t \leq 0.1$.

\begin{tabular}{lccr}
\hline$t$ & $\mathrm{VIM} u_{2}$ & $\mathrm{MVIM} u_{2}$ & Exact $u$ \\
\hline 0.0001 & -0.0009511410406405126 & -0.0009511426259533606 & $-0.9511426272 * 10^{-3}$ \\
0.001 & -0.0063049860784233505 & -0.0063060126254418855 & $-0.6306050198 * 10^{-2}$ \\
0.01 & -0.00998748230827825 & -0.010064948254214971 & $-0.1006914044 * 10^{-1}$ \\
0.1 & -0.009987964198100541 & -0.010893307586231409 & $-0.1096779217 * 10^{-1}$ \\
\hline
\end{tabular}

TABLE 12: Values for $v(t)$ for the second iteration by Variational Iteration Method (VIM) and Modified Variational Iteration Method (MVIM) compared with exact values of $v(t)$ in $0.0001 \leq t \leq 0.1$.

\begin{tabular}{lccr}
\hline$t$ & VIM $v_{2}$ & MVIM $v_{2}$ & Exact $v$ \\
\hline 0.0001 & $4.8355100352656666 * 10^{-8}$ & $4.835591010596274 * 10^{-8}$ & $0.4835591013 * 10^{-7}$ \\
0.001 & $3.6660943322046335 * 10^{-6}$ & $3.670267991501275 * 10^{-6}$ & $0.3670275606 * 10^{-5}$ \\
0.01 & 0.00008872285001837454 & 0.00008976891925882233 & $0.8978912350 * 10^{-4}$ \\
0.1 & 0.0009324013827985804 & 0.0009850573565074391 & $0.9879731668 * 10^{-3}$ \\
\hline
\end{tabular}

For $n=1$,

$$
\begin{aligned}
& m u_{2}(t)=m u_{1}(t) \\
& \quad-\int_{0}^{t} e^{-1011.01(s-t)} \\
& \quad \times\left[m u_{1}^{\prime}(s)-0.01\right. \\
& \quad+\left(0.01+m u_{1}(s)+m v_{1}(s)\right) \\
& \left.\quad \times\left(m u_{1}^{2}(t)+1001 m u_{1}(t)+1001\right)\right] d s,
\end{aligned}
$$

$$
m v_{2}(t)=m v_{1}(t)
$$

$$
\begin{aligned}
-\int_{0}^{t} e^{s-t} & {\left[m v_{1}^{\prime}(s)-0.01\right.} \\
& +\left(0.01+m u_{2}(s)+m v_{1}(s)\right) \\
& \left.\times\left(1+m v_{1}^{2}(s)\right)\right] d s
\end{aligned}
$$

or, as a computed result for this second iteration,

$$
\begin{aligned}
m u_{2}(t)= & -0.01968426729443655 \\
& +4.79046836800244 \\
& \times 10^{-10} e^{-3033.0299999999997 t}-\cdots, \\
m v_{2}(t)= & 0.01968424705929668 \\
& +9.090257649281869 \\
& \times 10^{-10} e^{-5055.049999999988 t}-\cdots .
\end{aligned}
$$

VIM and MVIM iterations of this problem are conducted up to 2 iterations. The results and related errors are given in Tables 11, 12, 13, and 14 for comparison purposes.
TABLE 13: Absolute Error Values for $u(t)$ for the second iteration by Variational Iteration Method (VIM) and Modified Variational Iteration Method (MVIM) in $0.0001 \leq t \leq 0.1$.

\begin{tabular}{lcc}
\hline$t$ & $\left|u(t)-u_{2}(t)\right|$ & $\left|u(t)-m u_{2}(t)\right|$ \\
\hline 0.0001 & $1.5865594874117303 * 10^{-9}$ & $1.2466394019514104 * 10^{-12}$ \\
0.001 & $1.0641195766500702 * 10^{-6}$ & $3.757255811510929 * 10^{-8}$ \\
0.01 & 0.0000816581317217499 & $4.192185785029806 * 10^{-6}$ \\
0.1 & 0.0009798279718994593 & 0.00007448458376859216 \\
\hline
\end{tabular}

TABLE 14: Absolute Error Values for $v(t)$ for the second iteration by Variational Iteration Method (VIM) and Modified Variational Iteration Method (MVIM) in $0.0001 \leq t \leq 0.1$.

\begin{tabular}{lcc}
\hline$t$ & $\left|v(t)-v_{2}(t)\right|$ & $\left|v(t)-m v_{2}(t)\right|$ \\
\hline 0.0001 & $8.097773433344856 * 10^{-13}$ & $2.4037259795860145 * 10^{-17}$ \\
0.001 & $4.181273795366942 * 10^{-9}$ & $3.757255811510929 * 10^{-8}$ \\
0.01 & $1.0662734816254679 * 10^{-6}$ & $2.0204241177684498 * 10^{-8}$ \\
0.1 & 0.00005557178400141969 & $2.915810292560949 * 10^{-6}$ \\
\hline
\end{tabular}

\section{Discussion and Conclusions}

In this research, we obtain numerical solutions for four examples of stiff systems of ordinary differential equations. In order to analyze the efficiency of the two methods, namely, VIM and MVIM, in comparison with exact solutions, the absolute errors are calculated for different $t$ values.

It can be seen that there are very good agreement between the results of VIM, results of MVIM, and exact solutions for these examples, which confirms the validity of the two methods for these problems. However, when solutions of VIM and MVIM are compared, it can be said that MVIM is providing better results in general.

These methods are very simple and do not require very small time steps for mildly stiff linear ordinary differential equations to obtain results in which error accumulation gets bigger as the time gets bigger for finding the solution. 


\section{References}

[1] A. S. Mahmood, L. Casasús, and W. Al-Hayani, "The decomposition method for stiff systems of ordinary differential equations," Applied Mathematics and Computation, vol. 167, no. 2, pp. 964-975, 2005.

[2] M. T. Darvishi, F. Khani, and A. A. Soliman, "The numerical simulation for stiff systems of ordinary differential equations," Computers \& Mathematics with Applications, vol. 54, no. 7-8, pp. 1055-1063, 2007.

[3] H. Aminikhah and M. Hemmatnezhad, "An effective modification of the homotopy perturbation method for stiff systems of ordinary differential equations," Applied Mathematics Letters, vol. 24, no. 9, pp. 1502-1508, 2011.

[4] A. S. Mahmood, L. Casasús, and W. Al-Hayani, "The decomposition method for stiff systems of ordinary differential equations," Applied Mathematics and Computation, vol. 167, no. 2, pp. 964-975, 2005.

[5] R. Alt, " $A$-stable one-step methods with step-size control for stiff systems of ordinary differential equations," Journal of Computational and Applied Mathematics, vol. 4, no. 1, pp. 2935, 1978.

[6] H. H. Rosenbrock and C. Storey, Computational Techniques For Chemical Engineers, Pergamon Press, New York, NY, USA, 1966.

[7] X.-Y. Wu and J.-L. Xia, "Two low accuracy methods for stiff systems," Applied Mathematics and Computation, vol. 123, no. 2, pp. 141-153, 2001.

[8] S. Abelman and K. C. Patidar, "Comparison of some recent numerical methods for initial-value problems for stiff ordinary differential equations," Computers \& Mathematics with Applications, vol. 55, no. 4, pp. 733-744, 2008.

[9] E. Hofer, "A partially implicit method for large stiff systems of ODEs with only few equations introducing small timeconstants," SIAM Journal on Numerical Analysis, vol. 13, no. 5, pp. 645-663, 1976.

[10] S. M. Hosseini and G. Hojjati, "Matrix free MEBDF method for the solution of stiff systems of ODEs," Mathematical and Computer Modelling, vol. 29, no. 4, pp. 67-77, 1999.

[11] J. C. Butcher, Numerical Methods for Ordinary Differential Equations, John Wiley \& Sons, New York, NY, USA, 2003.

[12] Ixaru, L. Gr, G. Vanden Berghe, and H. . De Meyer, "Frequency evaluation in exponential fitting multistep algorithms for ODEs," Journal of Computational and Applied Mathematics, vol. 140, pp. 423-434, 2000.

[13] L. Gr. Ixaru, G. Vanden Berghe, and H. De Meyer, "Exponentially fitted variable two-step BDF algorithms for first order ODEs," Computer Physics Communications, vol. 100, pp. 56-70, 2003.

[14] H. van de Vyver, "Two-step hybrid methods adapted to the numerical integration of perturbed oscillators," http://arxiv .org/abs/math/0612637.

[15] P. Kaps, "Rosenbrock-type methods," in Numerical Methods For Stiff Initial Value Problems. Berich, G. Dahlquist and R. Jeltsch, Eds., vol. 9, Inst. Fur Geometric und Practische Mathematik der RWTH Aachen, 1981.

[16] S. V. Fatunla, "An implicit two-point numerical integration formula for linear and nonlinear stiff systems of ordinary differential equations," Mathematics of Computation, vol. 32, no. 141, pp. 1-11, 1978.

[17] J. D. Lambert, Computational Methods in ODEs, John Wiley \& Sons, 1973.
[18] J. H. He, "Variational iteration method-a kind of non-linear analytical technique: some examples," International Journal of Non-Linear Mechanics, vol. 34, pp. 699-708, 1999.

[19] J. He, "A new approach to nonlinear partial differential equations," Communications in Nonlinear Science and Numerical Simulation, vol. 2, no. 4, pp. 230-235, 1997.

[20] J.-H. He, "Approximate analytical solution for seepage flow with fractional derivatives in porous media," Computer Methods in Applied Mechanics and Engineering, vol. 167, no. 1-2, pp. 27-68, 1998.

[21] J.-H. He, "Approximate solution of nonlinear differential equations with convolution product nonlinearities," Computer Methods in Applied Mechanics and Engineering, vol. 167, no. 1-2, pp. 69-73, 1998.

[22] J. H. He and X.-H. Wu, "Construction of solitary solution and compacton-like solution by variational iteration method," Chaos, Soliton and Fractals, vol. 29, pp. 108-113, 2006.

[23] J.-H. He, "Variational iteration method for autonomous ordinary differential systems," Applied Mathematics and Computation, vol. 114, no. 2-3, pp. 115-123, 2000.

[24] V. Marinca, "An approximate solution for one-dimensional weakly nonlinear oscillations," International Journal of Nonlinear Sciences and Numerical Simulation, vol. 3, no. 2, pp. 107-110, 2002.

[25] M. A. Abdou and A. A. Soliman, "New applications of variational iteration method," Physica D, vol. 211, no. 1-2, pp. 1-8, 2005.

[26] M. Inokuti, H. Sekine, and T. Mura, "General use of the Lagrange multiplier in nonlinear mathematics," in Variational Method in the Mechanics of Solids, Nemat-Nassers, Ed., pp. 156162, Pergamon Press, Oxford, UK, 1978.

[27] J. I. Ramos, "On the variational iteration method and other iterative techniques for nonlinear differential equations," Applied Mathematics and Computation, vol. 199, no. 1, pp. 39-69, 2008.

[28] J. H. He, G.-C. Wu, and F. Austin, "The variational iteration method which should be followed," Nonlinear Science Letters A, vol. 1, pp. 1-30, 2010.

[29] D. Kincaid and W. Cheney, Numerical Analysis-Mathematics of Scientific Computing, American Mathematical Society publications, 3rd edition, 2009.

[30] M. M. Stabrowski, "An efficient algorithm for solving stiff ordinary differential equations," Simulation Practice and Theory, vol. 5, no. 4, pp. 333-344, 1997. 


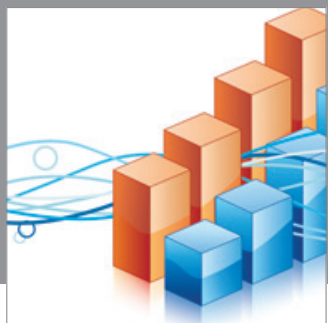

Advances in

Operations Research

mansans

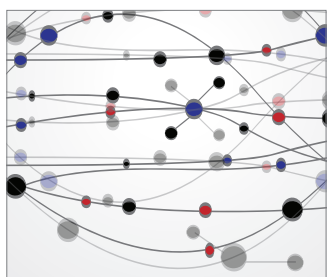

The Scientific World Journal
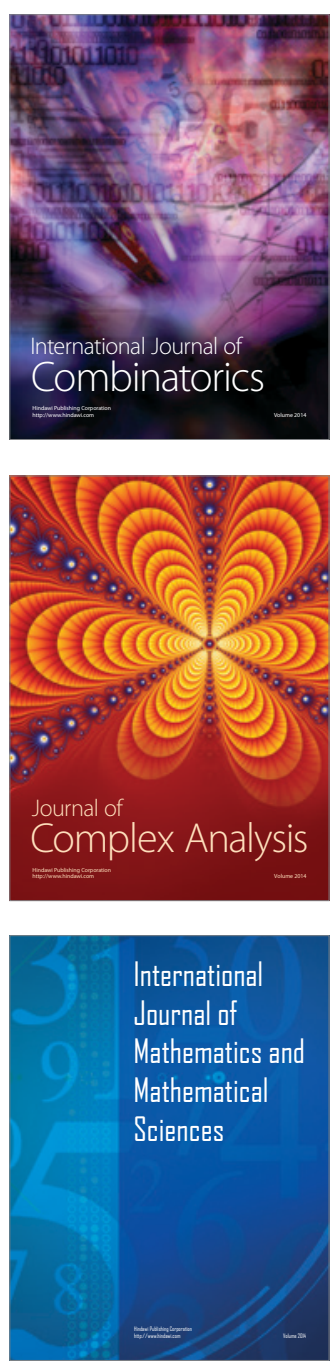
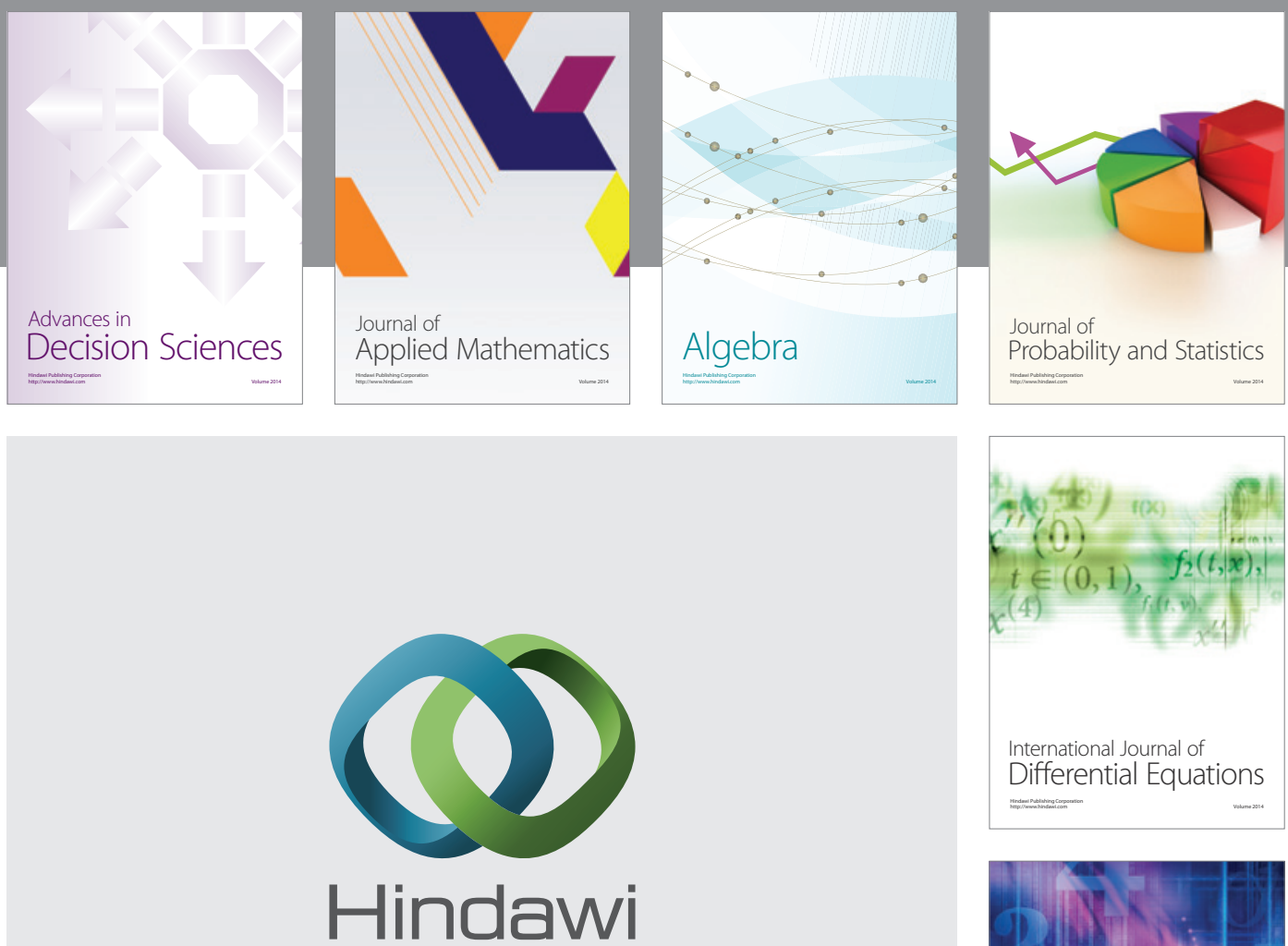

Submit your manuscripts at http://www.hindawi.com
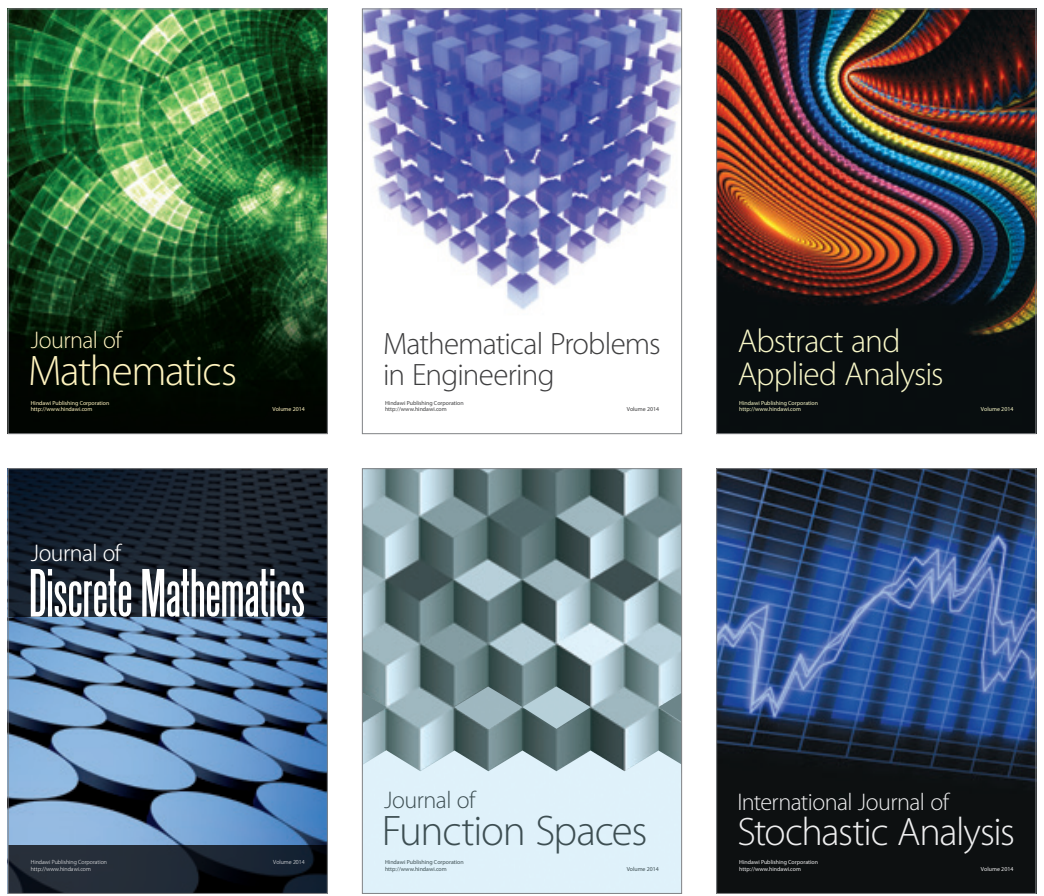

Journal of

Function Spaces

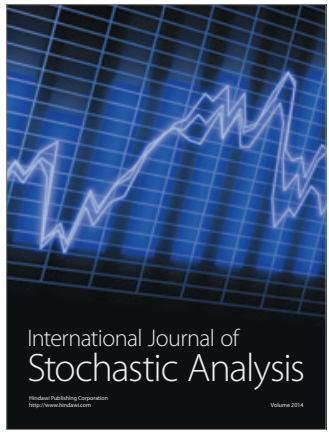

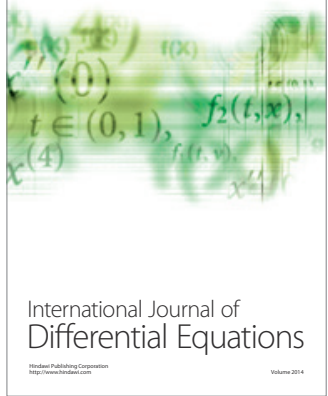
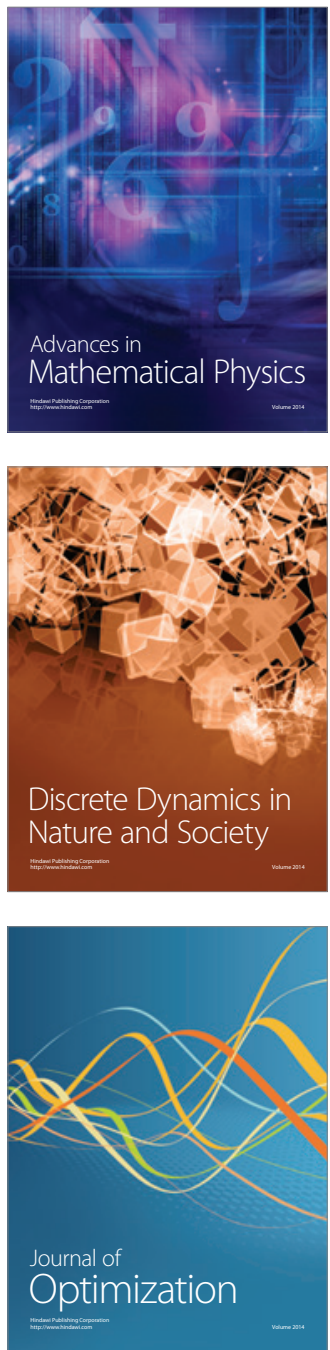